\title{
TS fuzzy controllers for cascaded systems*
}

\author{
Zs. Lendek, R. Babuška, and B. De Schutter
}

If you want to cite this report, please use the following reference instead: Zs. Lendek, R. Babuška, and B. De Schutter, "TS fuzzy controllers for cascaded systems," Proceedings of the 3rd IFAC Workshop on Advanced Fuzzy and Neural Control (AFNC 07), Valenciennes, France, pp. 43-48, Oct. 2007. Paper MO4-2.

Delft Center for Systems and Control Delft University of Technology

Mekelweg 2, 2628 CD Delft

The Netherlands

phone: +31-15-278.24.73 (secretary)

URL: https://www.dcsc.tudelft.nl

*This report can also be downloaded via https://pub.deschutter.info/abs/07_018.html 


\title{
TS FUZZY CONTROLLERS FOR CASCADED SYSTEMS
}

\author{
Zs. Lendek* R. Babuška* B. De Schutter*,** \\ * Delft Center for Systems and Control, Delft University of \\ Technology, Mekelweg 2, 2628 CD Delft, The Netherlands \\ $\{z s . l e n d e k, r . b a b u s k a\} @ t u d e l f t . n l$ \\ ** Marine and Transport Technology Department, Delft \\ University of Technology, b@deschutter.info
}

\begin{abstract}
A large class of dynamic systems can be decomposed into or approximated by cascaded subsystems. Applications include multi-agent systems, distributed process control, and hierarchical large-scale systems. Nonlinear dynamic systems can be represented as Takagi-Sugeno (TS) fuzzy models, with linear or affine consequents. For cascaded TS systems, it has been proven that the stability of the subsystems implies the stability of the overall system. In this paper, the cascaded approach is used for controller design. A theoretical design method for tracking TS controllers and a simulation example are presented. The results show that the distributed controller achieves the same performance as the centralized one, while leading to increased modularity, reduced complexity, lower computational costs, and easier tuning.
\end{abstract}

Keywords: fuzzy systems, fuzzy control, cascaded systems, Lyapunov stability.

\section{INTRODUCTION}

Traditionally, linear, time-invariant systems have dominated the control theory. The linearity and time-invariance make these types of systems easy to analyze. The main disadvantage is that such systems fail to describe nonlinear systems globally. An accurate approximation of a nonlinear system can only be expected in the vicinity of an equilibrium point.

A generic method for the design of a controller valid for all types of nonlinear systems has not been developed yet. However, Takagi-Sugeno fuzzy models (Takagi and Sugeno, 1985) can well approximate a large class of nonlinear systems (Fantuzzi and Rovatti, 1996).

The Takagi-Sugeno fuzzy model consists of a fuzzy rule base. The rule antecedents partition a given subspace of the model variables into fuzzy regions. The consequent of each rule is usually a linear or affine linear model, valid locally in the corresponding region.

It is well-known that the stability of these local models does not ensure the stability of the overall fuzzy model. Therefore, a range of stability conditions have been developed for Takagi-Sugeno fuzzy systems, most of them relying on the feasibility of an associated system of linear matrix inequalities (LMI) (Tanaka et al., 1998; Johansson et al., 1999; Bergsten et al., 2001).

Several types of controllers have been developed for Takagi-Sugeno fuzzy systems, of which the socalled parallel distributed compensators (PDCs) are the most well-known (Tanaka and Wang, 1997; Tanaka et al., 1998). In general, the design of controllers also leads to the feasibility problem of the associated LMIs. However, the complexity of the 
LMI problem grows exponentially with the number of antecedents and the stability analysis or synthesis problem eventually becomes intractable for a large number of rules.

An important class of distributed systems (material processing systems, chemical processes) can be represented as cascaded subsystems, leading to increased modularity and reduced complexity of the problem. In several cases, conclusions referring to the overall system can be drawn from the properties of the individual subsystems. Conditions to ensure overall stability of general cascades, based on the stability of nonlinear subsystems, were derived in (Sontag, 1989; Seibert and Suarez, 1990).

A special class of systems, represented as a TakagiSugeno fuzzy systems, which can be decomposed into cascaded subsystems is studied, and PDC controllers for the whole system based on the individual subsystems are designed. The idea behind this type of design is that many systems are cascaded (e.g., hierarchical large-scale systems), while others may be represented as cascaded, controllable subsystems, which are less complex than the original system. The main benefit of this approach is that it relaxes the conditions imposed by a global design. Global analysis may lead to infeasible LMI conditions, even if the system considered is stabilizable. More relaxed stability conditions are proposed, which may render the associated LMI problem feasible. Moreover, the dimension of the LMI problem is reduced.

The structure of the paper is as follows. Section 2 introduces the cascaded setting for nonlinear systems. Section 3 presents the stability conditions for cascaded TS fuzzy systems. The proposed controller design for cascaded fuzzy systems is presented in Section 4. An example is given in Section 5. Finally, Section 6 concludes the paper.

\section{PRELIMINARIES}

The main motivation to consider cascaded dynamical systems came from the analysis of the models obtained after input-output linearization. Thereafter, several stability conditions were derived for different types of subsystems. In this section, the general cascaded setting is presented.

\subsection{Cascaded Controllers}

Consider the following general, controllable nonlinear system:

$$
\begin{array}{cc}
\dot{x}_{1}=f_{1}(\boldsymbol{x}, \boldsymbol{u}) & y_{1}=h_{1}(\boldsymbol{x}, \boldsymbol{u}) \\
\dot{x}_{2}=f_{2}(\boldsymbol{x}, \boldsymbol{u}) & y_{2}=h_{2}(\boldsymbol{x}, \boldsymbol{u}) \\
\vdots & \vdots \\
\dot{x}_{n}=f_{n}(\boldsymbol{x}, \boldsymbol{u}) & y_{m}=h_{m}(\boldsymbol{x}, \boldsymbol{u})
\end{array}
$$

and assume that this system can be partitioned into controllable subsystems. For the ease of notation, only two subsystems are considered, without loss of generality:

$$
\dot{\boldsymbol{x}}_{1}=\boldsymbol{f}_{1}\left(\boldsymbol{x}_{1}, \boldsymbol{u}_{1}\right)
$$

and

$$
\dot{\boldsymbol{x}}_{2}=\boldsymbol{f}_{2}\left(\boldsymbol{x}_{1}, \boldsymbol{x}_{2}, \boldsymbol{u}_{1}, \boldsymbol{u}_{2}\right)
$$

so that (2) is controllable with $\boldsymbol{u}_{1}$ as control input and (3) is controllable with $\boldsymbol{u}_{2}$ as control input. In fact, for the subsystem (3), $\boldsymbol{x}_{1}$ and $\boldsymbol{u}_{1}$ are inputs.

In general, such a partition of the model does not necessarily exist. Moreover, if it exists, it might not be unique. If such a partition exists, controllers may be designed for the subsystems separately, with some controllers relying on the desired actions and states of the other controllers. For two subsystems, the cascaded controller structure is depicted in Figure 1, with $\boldsymbol{u}^{\mathrm{d}}$ and $\boldsymbol{x}^{\mathrm{d}}$ being the desired control signals and trajectories, respectively.

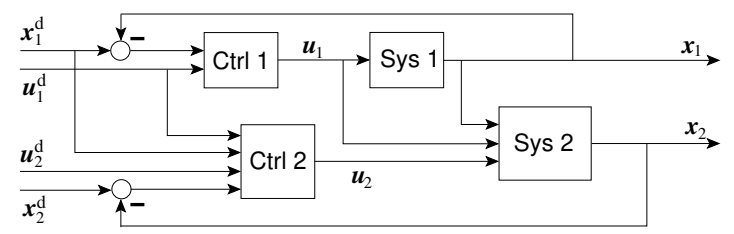

Fig. 1. Cascaded controllers.

\subsection{Cascaded Nonlinear Systems}

It is well-known that the cascade of stable linear systems is stable, since the eigenvalues of the joint system are determined only by the eigenvalues of the individual subsystems (Loria and Panteley, 2005). Therefore, the stability of the joint system is determined by the stability of the individual subsystems. However, the same reasoning does not necessarily hold for nonlinear or time-varying systems.

In the literature, the stability of several special cases has been studied. Beside the linear-nonlinear cascade (Arcak et al., 2002), more general cascades, in which both subsystems are nonlinear, were studied and conditions to ensure overall stability were derived in (Loria and Panteley, 2005).

According to these results, systems of the form:

$$
\begin{aligned}
& \dot{\boldsymbol{x}}_{1}=\boldsymbol{f}_{1}\left(\boldsymbol{x}_{1}\right) \\
& \dot{\boldsymbol{x}}_{2}=\boldsymbol{f}_{2}\left(\boldsymbol{x}_{2}\right)+\boldsymbol{g}\left(\boldsymbol{x}_{1}, \boldsymbol{x}_{2}\right)
\end{aligned}
$$

are globally asymptotically stable (GAS), assuming that the individual subsystems are GAS and, additionally, certain restrictions related to the continuity and/or slope apply for the interconnection term $\boldsymbol{g}$ (Jankovic et al., 1996; Chaillet and Loria, 2006). For particular conditions on the uniform global asymptotic stability of the cascaded system (4) see (Loria and Panteley, 2005). 


\section{CASCADED FUZZY SYSTEMS}

Consider an autonomous fuzzy system expressed as:

$$
\dot{\boldsymbol{x}}=\sum_{i=1}^{m} w_{i}(\boldsymbol{z}) A_{i} \boldsymbol{x}
$$

where $A_{i}, i=1,2, \ldots, m$ represents the $i$ th local linear model, $w_{i}$ is the corresponding normalized membership function, and $\boldsymbol{z}$ the vector of scheduling parameters. System (5) can also be regarded as a linear parameter varying (LPV) system:

$$
\dot{\boldsymbol{x}}=A(\boldsymbol{z}) \boldsymbol{x}
$$

with $A(\boldsymbol{z})=\sum_{i=1}^{m} w_{i}(\boldsymbol{z}) A_{i}$.

Consider now the case when the system matrices of the model (5) for each rule $i=1,2, \ldots, m$ are in a cascaded form. ${ }^{1}$ For the ease of notation, and without a loss of generality, only two subsystems are considered here:

$$
A_{i}=\left(\begin{array}{cc}
A_{1} & 0 \\
A_{21} & A_{2}
\end{array}\right)_{i}=\left(\begin{array}{cc}
A_{1 i} & 0 \\
A_{21 i} & A_{2 i}
\end{array}\right)
$$

i.e., system (5) can be expressed as the cascade of two fuzzy systems:

$$
\begin{aligned}
& \dot{\boldsymbol{x}}_{1}=\sum_{i=1}^{m} w_{1 i}\left(\boldsymbol{z}_{1}\right) A_{1 i} \boldsymbol{x}_{1} \\
& \dot{\boldsymbol{x}}_{2}=\sum_{i=1}^{m} w_{2 i}\left(\boldsymbol{z}_{1}, \boldsymbol{z}_{2}\right)\left(A_{21 i} \boldsymbol{x}_{1}+A_{2 i} \boldsymbol{x}_{2}\right)
\end{aligned}
$$

or, equivalently:

$$
\begin{aligned}
& \dot{\boldsymbol{x}}_{1}=A_{1}\left(\boldsymbol{z}_{1}\right) \boldsymbol{x}_{1} \\
& \dot{\boldsymbol{x}}_{2}=A_{21}\left(\boldsymbol{z}_{1}, \boldsymbol{z}_{2}\right) \boldsymbol{x}_{1}+A_{2}\left(\boldsymbol{z}_{1}, \boldsymbol{z}_{2}\right) \boldsymbol{x}_{2}
\end{aligned}
$$

with $A_{1}\left(\boldsymbol{z}_{1}\right)=\sum_{i=1}^{m} w_{1 i}\left(\boldsymbol{z}_{1}\right) A_{1 i}$, etc.

Consider now the subsystems

$$
\dot{\boldsymbol{x}}_{1}=A_{1}\left(\boldsymbol{z}_{1}\right) \boldsymbol{x}_{1}
$$

and

$$
\dot{\boldsymbol{x}}_{2}=A_{2}\left(\boldsymbol{z}_{1}, \boldsymbol{z}_{2}\right) \boldsymbol{x}_{2}
$$

It has been proven (Lendek et al., 2007), that

Theorem 1. If there exist two Lyapunov functions of the form $V_{1}\left(\boldsymbol{x}_{1}\right)=\boldsymbol{x}_{1}^{T} P_{1} \boldsymbol{x}_{1}$ and $V_{2}\left(\boldsymbol{x}_{2}\right)=$ $\boldsymbol{x}_{2}^{T} P_{2} \boldsymbol{x}_{2}$ so that the subsystems (9) and (10) are UGAS, then the cascaded system (8) is also $U G A S$.

Therefore, the uniform global asymptotic stability of the subsystems implies the stability of the global system. To prove the stability of the subsystems, known methods can be used, such as (Tanaka et al., 1998; Johansson et al., 1999).

1 If the fuzzy system is an approximation of a general nonlinear system, then this requires that the original nonlinear system is cascadable.

\section{CASCADED FUZZY CONTROLLERS}

\subsection{State Feedback Fuzzy Control}

Consider the TS system with affine consequents:

$$
\dot{\boldsymbol{x}}=\sum_{i=1}^{m} w_{i}(\boldsymbol{z})\left(A_{i} \boldsymbol{x}+B_{i} \boldsymbol{u}+a_{i}\right)
$$

or, equivalently,

$$
\dot{\boldsymbol{x}}=A(\boldsymbol{z}) \boldsymbol{x}+B(\boldsymbol{z}) \boldsymbol{u}+a(\boldsymbol{z})
$$

In general, the error state feedback control law has the form (Bergsten, 2001)

$$
\boldsymbol{u}=\boldsymbol{u}^{\mathrm{d}}+K(\boldsymbol{z})\left(\boldsymbol{x}-\boldsymbol{x}^{\mathrm{d}}\right)
$$

with $K(\boldsymbol{z})=\sum_{i=1}^{m} w_{i}(\boldsymbol{z}) K_{i}$, and the desired input signal $\boldsymbol{u}^{\mathrm{d}}$ computed so that the motion of the system is the desired $\boldsymbol{x}^{\mathrm{d}}$.

4.1.1. Fuzzy Regulators Consider system (12). The control goal is to bring the system to an equilibrium state. The control law has the form (13), with $\boldsymbol{u}^{\mathrm{d}}$ chosen so that $B(\boldsymbol{z}) \boldsymbol{u}^{\mathrm{d}}$ compensates the affine term $a(\boldsymbol{z})$. Then, the tracking error of the closed-loop system can be expressed as:

$$
\dot{\boldsymbol{e}}=\sum_{i=1}^{m} \sum_{j=1}^{m} w_{i}(\boldsymbol{z}) w_{j}(\boldsymbol{z})\left(A_{i}+B_{i} K_{j}\right) \boldsymbol{e}
$$

for which several LMI-based stability conditions have been derived (Tanaka et al., 1998).

4.1.2. Fuzzy Tracking Controllers In this case, the control goal is to track a desired trajectory $\boldsymbol{x}^{\mathrm{d}}$. Therefore, the desired input signal $\boldsymbol{u}^{\mathrm{d}}$ is computed so that the motion of the system is the desired one, i.e.,:

$$
\dot{\boldsymbol{x}}^{\mathrm{d}}=A\left(\boldsymbol{z}^{\prime}\right) \boldsymbol{x}^{\mathrm{d}}+B\left(\boldsymbol{z}^{\prime}\right) \boldsymbol{u}^{\mathrm{d}}+a\left(\boldsymbol{z}^{\prime}\right)
$$

$\boldsymbol{z}^{\prime}$ is the scheduling vector of the trajectory generator, which may differ from $\boldsymbol{z}$. Defining the tracking error $\boldsymbol{e}=\boldsymbol{x}-\boldsymbol{x}^{\mathrm{d}}, \dot{\boldsymbol{e}}=\dot{\boldsymbol{x}}-\dot{\boldsymbol{x}}^{\mathrm{d}}$, the closedloop error system can be written as:

$$
\begin{aligned}
\dot{\boldsymbol{e}}=\sum_{i=1}^{m} & \sum_{j=1}^{m} w_{i}(\boldsymbol{z}) w_{j}(\boldsymbol{z})\left(A_{i}+B_{i} K_{j}\right) \boldsymbol{e} \\
& -\dot{\boldsymbol{x}}^{\mathrm{d}}+A\left(\boldsymbol{z}^{\prime}\right) \boldsymbol{x}^{\mathrm{d}}+B\left(\boldsymbol{z}^{\prime}\right) \boldsymbol{u}^{\mathrm{d}}+a\left(\boldsymbol{z}^{\prime}\right)
\end{aligned}
$$

Here, $\dot{\boldsymbol{x}}^{\mathrm{d}}, \boldsymbol{x}^{\mathrm{d}}$ and $\boldsymbol{u}^{\mathrm{d}}$ are obtained by using a trajectory generator (Bergsten, 2001) so that the term $-\dot{\boldsymbol{x}}^{\mathrm{d}}+A\left(\boldsymbol{z}^{\prime}\right) \boldsymbol{x}^{\mathrm{d}}+B\left(\boldsymbol{z}^{\prime}\right) \boldsymbol{u}^{\mathrm{d}}+a\left(\boldsymbol{z}^{\prime}\right) \rightarrow 0$ as $\boldsymbol{x} \rightarrow \boldsymbol{x}^{\mathrm{d}}$. Therefore, it has to be ensured that the error system

$$
\dot{\boldsymbol{e}}=\sum_{i=1}^{m} \sum_{j=1}^{m} w_{i}(\boldsymbol{z}) w_{j}(\boldsymbol{z})\left(A_{i}+B_{i} K_{j}\right) \boldsymbol{e}
$$

is stable. Well-known conditions for this have been formalized in (Tanaka et al., 1998; Taniguchi et al., 1999; Wang et al., 2000). 


\subsection{Cascaded Fuzzy Control}

As before, consider the fuzzy system (12) and a fuzzy controller of the form (13). For the ease of notation, only two subsystems are considered, without a loss of generality. Then, the system matrices for each rule $i=1,2, \ldots, m$ can be written as:

$$
\begin{aligned}
A_{i} & =\left(\begin{array}{cc}
A_{1} & 0 \\
A_{21} & A_{2}
\end{array}\right)_{i}=\left(\begin{array}{cc}
A_{1 i} & 0 \\
A_{21 i} & A_{2 i}
\end{array}\right) \\
B_{i} & =\left(\begin{array}{cc}
B_{1} & 0 \\
B_{21} & B_{2}
\end{array}\right)_{i}=\left(\begin{array}{cc}
B_{1 i} & 0 \\
B_{21 i} & B_{2 i}
\end{array}\right)
\end{aligned}
$$

and the controllers can be designed individually for each subsystem and each rule, with the overall controller gain having the form $K_{i}=\left(\begin{array}{cc}K_{1 i} & 0 \\ 0 & K_{2 i}\end{array}\right)$, where $i$ denotes the rule number.

\subsubsection{Cascaded Fuzzy Regulators} cascaded fuzzy system of the form:

Consider a

$$
\begin{aligned}
& \dot{\boldsymbol{x}}_{1}=\sum_{i=1}^{m} w_{1 i}\left(\boldsymbol{z}_{1}\right)\left(A_{1 i} \boldsymbol{x}_{1}+B_{1 i} \boldsymbol{u}_{1}\right) \\
& \dot{\boldsymbol{x}}_{2}=\sum_{i=1}^{m} w_{2 i}\left(\boldsymbol{z}_{1}, \boldsymbol{z}_{2}\right)\left(A_{21 i} \boldsymbol{x}_{1}+A_{2 i} \boldsymbol{x}_{2}+B_{21 i} \boldsymbol{u}_{1}+B_{2 i} \boldsymbol{u}_{2}\right)
\end{aligned}
$$

Note that for affine systems, the control input is chosen so that it compensates the affine terms. Then, applying the state feedback controls $\boldsymbol{u}_{1}=$ $K_{1}\left(\boldsymbol{z}_{1}\right) \boldsymbol{x}_{1}$ and $\boldsymbol{u}_{2}=K_{2}(\boldsymbol{z}) \boldsymbol{x}_{2}$, to the subsystems

$$
\dot{\boldsymbol{x}}_{1}=A_{1}\left(\boldsymbol{z}_{1}\right) \boldsymbol{x}_{1}+B_{1}\left(\boldsymbol{z}_{1}\right) \boldsymbol{u}_{1}
$$

and

$$
\dot{\boldsymbol{x}}_{2}=A_{2}(\boldsymbol{z}) \boldsymbol{x}_{2}+B_{2}(\boldsymbol{z}) \boldsymbol{u}_{2}
$$

respectively, the error of the closed-loop system has the form:

$$
\begin{aligned}
& \dot{\boldsymbol{e}}=\sum_{i=1}^{m} \sum_{j=1}^{m} w_{i}(\boldsymbol{z}) w_{j}(\boldsymbol{z})\left(A_{i}+B_{i} K_{j}\right) \boldsymbol{e} \\
& =\sum_{i=1}^{m} \sum_{j=1}^{m} w_{i}(\boldsymbol{z}) w_{j}(\boldsymbol{z})\left(\begin{array}{cc}
A_{1}+B_{1} K_{1} & 0 \\
A_{21}+B_{21} K_{1} & A_{2}+B_{2} K_{2}
\end{array}\right)_{i j} \boldsymbol{e} .
\end{aligned}
$$

System (21) is also cascaded, and, based on Theorem 1 , it is stable, if the independent subsystems are stable, i.e., the control laws $\boldsymbol{u}_{1}=K_{1}\left(\boldsymbol{z}_{1}\right) \boldsymbol{x}_{1}$ and $\boldsymbol{u}_{2}=K_{2}(\boldsymbol{z}) \boldsymbol{x}_{2}$ stabilize the individual subsystems. These conditions can be formalized as:

Theorem 2. The system (21) is asymptotically stable, if there exist $P_{1}=P_{1}^{T}>0$ and $P_{2}=P_{2}^{T}>$ 0 , so that

$$
\begin{aligned}
& \left(A_{1 i}+B_{1 i} K_{1 i}\right)^{T} P_{1}+P_{1}\left(A_{1 i}+B_{1 i} K_{1 i}\right)<0 \\
& \left(A_{2 i}+B_{2 i} K_{2 i}\right)^{T} P_{2}+P_{2}\left(A_{2 i}+B_{2 i} K_{2 i}\right)<0 \\
& G_{1 i j}^{T} P_{1}+P_{1} G_{1 i j} \leq 0 \quad \forall w_{1 i}\left(\boldsymbol{z}_{1}\right) w_{1 j}\left(\boldsymbol{z}_{1}\right)>0 \\
& G_{2 i j}^{T} P_{2}+P_{2} G_{2 i j} \leq 0 \quad \forall w_{2 i}(\boldsymbol{z}) w_{2 j}(\boldsymbol{z})>0 \\
& G_{1 i j}=A_{1 i}+B_{1 i} K_{1 j}+A_{1 j}+B_{1 j} K_{1 i} \\
& G_{2 i j}=A_{2 i}+B_{2 i} K_{2 j}+A_{2 j}+B_{2 j} K_{2 i}
\end{aligned}
$$

Proof: Since system (21) is cascaded, based on Theorem 1, the stability of the individual subsystems $\dot{\boldsymbol{e}}_{1}=\sum_{i=1}^{m} \sum_{j=1}^{m} w_{i}(\boldsymbol{z}) w_{j}(\boldsymbol{z})\left(A_{1}+\right.$ $\left.B_{1} K_{1}\right)_{i j} \boldsymbol{e}_{1}$ and $\dot{\boldsymbol{e}}_{2}=\sum_{i=1}^{m} \sum_{j=1}^{m} w_{i}(\boldsymbol{z}) w_{j}(\boldsymbol{z})\left(A_{2}+\right.$ $\left.B_{2} K_{2}\right)_{i j} \boldsymbol{e}_{2}$ implies the stability of the cascade. The stability of a regulated system is ensured if there exists $P=P^{T}>0$ so that (Tanaka et al., 1998):

$$
\begin{aligned}
& \left(A_{i}+B_{i} K_{i}\right)^{T} P+P\left(A_{i}+B_{i} K_{i}\right)<0 \\
& G_{i j}^{T} P+P G_{i j} \leq 0 \quad \forall w_{i}(\boldsymbol{z}) w_{j}(\boldsymbol{z})>0 \\
& G_{i j}=A_{i}+B_{i} K_{j}+A_{j}+B_{j} K_{i}
\end{aligned}
$$

Applying the above conditions to both subsystems, the conditions (22) are obtained.

4.2.2. Cascaded Fuzzy Tracking Controllers For tracking, the control laws have the form:

$$
\boldsymbol{u}_{1}=\boldsymbol{u}_{1}^{\mathrm{d}}+K_{1}\left(\boldsymbol{z}_{1}\right)\left(\boldsymbol{x}_{1}-\boldsymbol{x}_{1}^{\mathrm{d}}\right)
$$

for the first subsystem and

$$
\boldsymbol{u}_{2}=\boldsymbol{u}_{2}^{\mathrm{d}}+K_{2}(\boldsymbol{z})\left(\boldsymbol{x}_{2}-\boldsymbol{x}_{2}^{\mathrm{d}}\right)
$$

for the second subsystem. The error system can be written as:

$$
\begin{aligned}
\dot{\boldsymbol{e}}= & \sum_{i=1}^{m} \sum_{j=1}^{m} w_{i}(\boldsymbol{z}) w_{j}(\boldsymbol{z})\left(A_{i}+B_{i} K_{j}\right) \boldsymbol{e} \\
& -\dot{\boldsymbol{x}}^{\mathrm{d}}+\left(\begin{array}{cc}
A_{1}\left(\boldsymbol{z}_{1}\right) & 0 \\
A_{21}(\boldsymbol{z}) & A_{2}(\boldsymbol{z})
\end{array}\right)\left(\begin{array}{c}
\boldsymbol{x}_{1}^{\mathrm{d}} \\
\boldsymbol{x}_{2}^{\mathrm{d}}
\end{array}\right) \\
& +\left(\begin{array}{cc}
B_{1}\left(\boldsymbol{z}_{1}\right) & 0 \\
B_{21}(\boldsymbol{z}) & B_{2}(\boldsymbol{z})
\end{array}\right)\left(\begin{array}{c}
\boldsymbol{u}_{1}^{\mathrm{d}} \\
\boldsymbol{u}_{2}^{\mathrm{d}}
\end{array}\right)+\left(\begin{array}{c}
a_{1}\left(\boldsymbol{z}_{1}\right) \\
a_{2}(\boldsymbol{z})
\end{array}\right)
\end{aligned}
$$

From Section 4.2.1, if the control laws (23) and (24) stabilize the individual subsystems, the error system itself (without the desired trajectory) is asymptotically stable. Therefore, the behavior of the closed-loop system is only influenced by the desired trajectory. To generate the desired input $\boldsymbol{u}_{2}^{\mathrm{d}}$ and state trajectory $\boldsymbol{x}_{2}^{\mathrm{d}}$ of the second subsystem (18), the state trajectory and input trajectory of the first subsystem must be taken into account. The trajectories are generated so that:

$$
\begin{gathered}
\dot{\boldsymbol{x}}_{1}^{\mathrm{d}}=A_{1}\left(\boldsymbol{z}_{1}\right) \boldsymbol{x}_{1}^{\mathrm{d}}+B_{1}\left(\boldsymbol{z}_{1}\right) \boldsymbol{u}_{1}^{\mathrm{d}}+a_{1}\left(\boldsymbol{z}_{1}\right) \\
\dot{\boldsymbol{x}}_{2}^{\mathrm{d}}=A_{2}(\boldsymbol{z}) \boldsymbol{x}_{2}^{\mathrm{d}}+B_{2}\left(\boldsymbol{z}_{1}\right) \boldsymbol{u}_{2}^{\mathrm{d}}+a_{2}(\boldsymbol{z}) \\
+A_{21}(\boldsymbol{z}) \boldsymbol{x}_{1}^{\mathrm{d}}+B_{21}(\boldsymbol{z}) \boldsymbol{u}_{2}^{\mathrm{d}}
\end{gathered}
$$

Note that, since the dimension of the problem is reduced, generating such trajectories is less complex than generating trajectories for the centralized system. Moreover, if the desired trajectories are piecewise constant, the problem is equivalent to solving the equations:

$$
\begin{aligned}
& 0=A_{1}\left(\boldsymbol{z}_{1}\right) \boldsymbol{x}_{1}^{\mathrm{d}}+B_{1}\left(\boldsymbol{z}_{1}\right) \boldsymbol{u}_{1}^{\mathrm{d}}+a_{1}\left(\boldsymbol{z}_{1}\right) \\
& 0=A_{2}(\boldsymbol{z}) \boldsymbol{x}_{2}^{\mathrm{d}}+B_{2}(\boldsymbol{z}) \boldsymbol{u}_{2}^{\mathrm{d}}+a_{2}^{1}(\boldsymbol{z})
\end{aligned}
$$

where $a_{2}^{1}(\boldsymbol{z})=A_{21}(\boldsymbol{z}) \boldsymbol{x}_{1}^{\mathrm{d}}+B_{21}(\boldsymbol{z}) \boldsymbol{u}_{2}^{\mathrm{d}}+a_{2}(\boldsymbol{z})$. 


\section{EXAMPLE}

Consider the two tanks system as shown in Figure 2. Water is pumped from a reservoir into the upper tank (1). From this tank, the water flows to the lower tanks and then back to the reservoir. The system has two control inputs $u_{1}$ and $u_{2}$, which are the voltages applied to the motors of the pumps. It is assumed that both flow rates $\left(F_{\text {in,1 }}\right.$ and $\left.F_{\text {in, } 2}\right)$ and the levels in the tanks $\left(h_{1}\right.$ and $\left.h_{2}\right)$ are measured. The goal is to control the levels in the tanks. The equations describing the dynamics are:

$$
\begin{aligned}
\tau \dot{F}_{\mathrm{in}, 1} & =-F_{\mathrm{in}, 1}+Q_{s, 1} \cdot u_{1} \\
\dot{h}_{1} & =\frac{F_{\mathrm{in}, 1}}{A_{1}}-\frac{s_{1} \sqrt{2 g h_{1}}}{A_{1}} \\
\tau \dot{F}_{i n, 2} & =-F_{\mathrm{in}, 2}+Q_{s, 2} \cdot u_{2} \\
\dot{h}_{2} & =\frac{s_{1} \sqrt{2 g h_{1}}}{A_{2}}-\frac{s_{2} \sqrt{2 g h_{2}}}{A_{2}}+\frac{F_{\mathrm{in}, 2}}{A_{2}} \\
&
\end{aligned}
$$

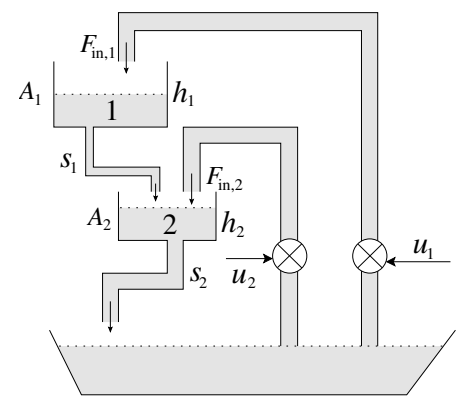

Fig. 2. Cascaded tanks system.

The parameter values are presented in Table 1 .

Table 1. Parameter values used.

\begin{tabular}{llll} 
Parameter & Symbol & Value & Units \\
\hline Acceleration & $g$ & 981 & $\mathrm{~cm} / \mathrm{s}^{2}$ \\
Area of the tanks & $A_{1}, A_{2}$ & 10,9 & $\mathrm{~cm}^{2}$ \\
Outlet area of tanks & $s_{1}, s_{2}$ & $0.1,0.2$ & $\mathrm{~cm}^{2}$ \\
Input to flow gains & $Q_{s, 1}, Q_{s, 2}$ & $5,33.3$ & $\mathrm{~cm}^{3} / \mathrm{s} / \mathrm{V}$ \\
Motor time constants & $\tau_{1}, \tau_{2}$ & $0.1,3$ & $\mathrm{~s}$ \\
\hline
\end{tabular}

It is assumed that the tanks have the same height, $h_{\max }=2 \mathrm{~cm}$. Therefore, all levels are bounded, $h_{i} \in\left[0, h_{\max }\right]$. In order to use the proposed design, a TS fuzzy model of the system (26) is constructed. For each level $h_{i}$, four points $h_{i} \in$ $\{0.1,0.55,1.05,1.6\}$ are chosen, together with appropriate membership functions, depicted in Figure 3.

The system (26) is linearized for each combination of the chosen points. The scheduling vector consists of the heights $h_{1}$ and $h_{2}$, which are the controlled states. Since the linearization is not done in equilibrium points, the consequents are affine. For instance, the rule obtained by linearizing in $h_{1}=1.05, h_{2}=0.55$ has the following consequents:

$$
\begin{aligned}
A & =\left(\begin{array}{cccc}
-10 & 0 & 0 & 0 \\
0.1 & -0.22 & 0 & 0 \\
0 & 0 & -0.33 & 0 \\
0 & 0.22 & 0.1 & -0.60
\end{array}\right) \quad B=\left(\begin{array}{cc}
50 & 0 \\
0 & 0 \\
0 & 11.1 \\
0 & 0
\end{array}\right) \\
a & =\left(\begin{array}{lll}
0-0.23 & 0-0.1)^{T}
\end{array}\right.
\end{aligned}
$$

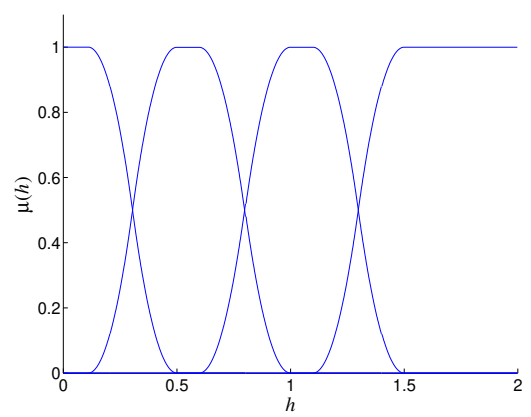

Fig. 3. Membership functions for the heights.

By examining the form of the system (26) or the consequent state matrices, one can easily see that the system can be cascaded, with $\boldsymbol{x}_{1}=\left[\begin{array}{ll}F_{\text {in,1 }} & h_{1}\end{array}\right]^{T}$ and $\boldsymbol{x}_{2}=\left[\begin{array}{ll}F_{\mathrm{in}, 2} & h_{2}\end{array}\right]^{T}$. Therefore, controllers can be designed separately for the individual subsystems. Controllers are designed both for the whole system and the cascaded subsystems using the same pole placement method and conditions. Both controllers have the form (13).

The controllers are applied to the fuzzy system. The same trajectory is tracked in both cases. To simulate the system, first the equations were discretized with the Euler method, using a sampling period $T=0.05 \mathrm{~s}$. First the desired inputs were randomly generated, from which the desired states were computed. The resulting states are nonstationary. The closed-loop error for the cascaded and centralized control is presented in Figure 4. As can be seen, the performance of the controllers is comparable.
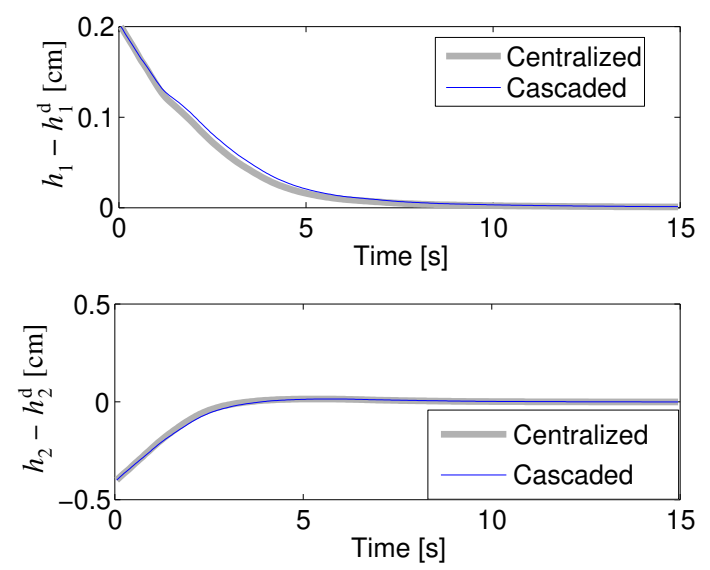

Fig. 4. Closed-loop errors for $h_{1}$ and $h_{2}$ with centralized and cascaded controllers.

A better approximation of system (26) can be obtained by linearizing it closer to 0 (e.g., at 
$h_{1}=h_{2}=0.01$, instead of 0.1 ). In this case, the centralized controller cannot track the desired trajectories. However, the cascaded controller still tracks them. The error for the centralized and cascaded controller for $h_{2}$ can be seen in Figure 5 . The cascaded approach is also less demanding in terms of the CPU time needed to solve the LMI problems for computing the controller $(0.125 \mathrm{~s}$ for the cascaded, $0.485 \mathrm{~s}$ for the centralized controller).
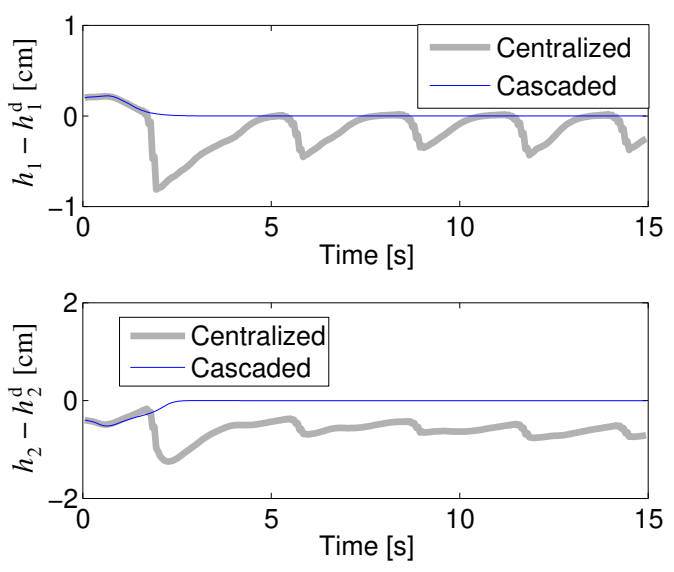

Fig. 5. Closed-loop error for $h_{1}$ and $h_{2}$. The centralized controller cannot track the desired trajectories.

\section{CONCLUSIONS}

In many real-life applications, a complex process model can be decomposed into simpler, cascaded subsystems. This partitioning of a process leads to increased modularity and reduced complexity of the problem, while also making the analysis easier. In this paper, the cascaded setting has been used for controller design. If a complex process model can be decomposed in simpler subsystems, controllers can be designed for these individual subsystems. This partitioning of a process and controller leads to increased modularity and reduced complexity of the problem, with reduced computational costs. The benefits of this approach have been demonstrated on a simulation example.

Acknowledgment Research sponsored by Senter, Ministry of Economic Affairs of the Netherlands within project Interactive Collaborative Information Systems (grant BSIK03024).

\section{REFERENCES}

Arcak, M., D. Angeli and E.D. Sontag (2002). A unifying integral ISS framework for stability of nonlinear cascades. SIAM J. Control Optim. 40, 1888-1904.
Bergsten, P. (2001). Observers and controllers for Takagi-Sugeno fuzzy systems. PhD thesis. Örebro University.

Bergsten, P., R. Palm and D. Driankov (2001). Fuzzy observers. In: 10th IEEE Int. Conf. on Fuzzy Systems, pp. 700-703.

Chaillet, A. and A. Loria (2006). Necessary and sufficient conditions for uniform semiglobal practical asymptotic stability: Application to cascaded systems. Automatica 42, 1899-1906.

Fantuzzi, C. and R. Rovatti (1996). On the approximation capabilities of the homogeneous TS model. In: 5th IEEE Int. Conf. on Fuzzy Systems. pp. 1067-1072.

Jankovic, M., R. Sepulchre and P.V. Kokotovic (1996). Constructive Lyapunov stabilization of nonlinear cascade systems. IEEE Trans. on Automatic Control 41, 1723 - 1735.

Johansson, M., A. Rantzer and K.E. Arzen (1999). Piecewise quadratic stability of fuzzy systems. IEEE Trans. on Fuzzy Systems 7, 713722 .

Lendek, Zs., R. Babuška and B. De Schutter (2007). Stability of cascaded Takagi-Sugeno fuzzy systems. Accepted for FUZZ-IEEE 2007.

Loria, A. and E. Panteley (2005). Advanced Topics in Control Systems Theory. Chap. Cascaded nonlinear time-varying systems: Analysis and design, pp. 23-64. Springer.

Seibert, P. and R. Suarez (1990). Global stabilization of nonlinear cascade systems. Systems $\mathbb{E}$ Control Letters 14, 347-352.

Sontag, E. D. (1989). Smooth stabilization implies coprime factorization. IEEE Trans.on Automatic Control, 34, 435-443.

Takagi, T. and M. Sugeno (1985). Fuzzy identification of systems and its applications to modeling and control. IEEE Trans. on Systems, Man, and Cybernetics 15, 116-132.

Tanaka, K. and H.O. Wang (1997). Fuzzy regulators and fuzzy observers: a linear matrix inequality approach. In: 36th IEEE Conf. on Decision and Control. Vol. 2. pp. $1315-1320$.

Tanaka, K., T. Ikeda and H.O. Wang (1998). Fuzzy regulators and fuzzy observers: relaxed stability conditions and LMI-based designs. IEEE Trans. on Fuzzy Systems 6, 250-265.

Taniguchi, T., K. Tanaka, K. Yamafuji and H.O. Wang (1999). Nonlinear model following control via Takagi-Sugeno fuzzy model. In: American Control Conf., pp. 1837-1841.

Wang, H.O., J. Li, D. Niemann and K. Tanaka (2000). T-S fuzzy model with linear rule consequence and PDC controller: a universal framework for nonlinear control systems. In: 9th IEEE Int. Conf. on Fuzzy Systems, pp. 549-554. 